\title{
Preschool teachers' preparation programs in Turkey: A multi-level analysis of variables on classroom management models
}

\author{
Türkiye'deki okul öncesi öğretmenliği hazırlık programları: Sınıf yönetimi \\ modellerinde değişkenlerin çok düzeyli analizi \\ Abdulhamit Karademir ${ }^{1}$, Özkan Saatçioğlu ${ }^{2}$
}

\section{Article History}

Received : 04 January 2021

Accepted : 08 April 2021

\section{Article Type}

Research Article

\begin{abstract}
This quantitative study aimed to address variables related to six classroom management models used by 1323 preservice preschool teachers: behavioral change theory, Dreikurs, Canter, Glasser, Kounin, and Gordon models. Data were collected using a demographic characteristics form and the Classroom Management Strategy Determination Scale. Data were analyzed using hierarchical linear modeling. Results showed that the classroom management course taught by experts helped preservice preschool teachers choose the right models. Gender played a role in Dreikurs' social discipline model, while undergraduate education played a role in Canter's assertive discipline model.
\end{abstract}

Keywords: Early childhood education, Undesired behaviors, Classroom management models, Preservice teachers

Öz: Bu nicel araştırma 1323 okul öncesi öğretmen adayının tercih ettiği sınıf yönetimi modelleriyle ilişkili değişkenleri şu altı disiplin modeli bağlamında araștırmaktadır: Behavioral change theory, Dreikurs, Canter, Glasser, Kounin ve Gordon modeli. Veriler demografik bilgi formu ve Classroom Management Strategy Determination Scale ile toplanmıştır. Öğrenci ve üniversite düzeyindeki değisskenlerin HLM ile analiz edilmesi sonucunda sınıf yönetimi dersinin, alan uzmanları tarafindan yürütülmesinin öğretmen adaylarının doğru ve etkili modelleri belirlemeleri üzerinde etkili olduğu anlaşılmıştır. Bununla birlikte Dreikurs sosyal disiplin modeli için cinsiyetin, Canter modeli için ise önceden lisans eğitimi almanın etkili olduğu bulunmuştur.

Anahtar Kelimeler: Erken çocukluk eğitimi, İstenmeyen davranışlar, Sınıf yönetimi modelleri, Öğretmen adayları 


\section{INTRODUCTION}

Today's preschool approaches offer free and creative educational environments, promote highlevel learning, make children feel valued, and encourage them to take risks and develop selfregulation skills (Buyse et al., 2008; Hamre et al., 2012; Mikami et al., 2012). Well-designed educational environments help reduce the prevalence of undesired behavior (Neal, Norwalk \& Haskett, 2020), make children more interested in learning, and provide an opportunity for effective interaction (Bank, 2014; Lippard, La Paro, Rouse \& Crosby, 2018). Education is a continuous process, and therefore, behavioral and developmental problems should be resolved at an early age for high academic performance (Gettinger \& Fischer, 2014). Diagnosing undesired behaviors at an early age helps children turn them into desired ones in the future (Sun, 2015; Yumuş \& Bayhan, 2017). Instructors are vigilant about undesired behavior in educational settings because it affects classroom management (Emmer \& Stough, 2001). Undesired behavior is defined as any behavior that disrupts the learning atmosphere, teacher-student interaction, and class flow (Beaman, Wheldall \& Kemp, 2007).

\section{Classroom management models}

Teachers with the right classroom management strategies are more likely to achieve targeted learning outcomes (Emmer \& Stough, 2001). Teachers implement classroom management models and strategies to eliminate undesired behavior (Martin \& Sass, 2010; Şahin-Sak, İ. T., Sak, R., \& Tezel-Şahin, 2018). Such models help teachers manage the class and grant students some rights. The tighter the classroom control, the more stringent the rules and their consequences. On the contrary, the looser the classroom control, the more responsible the students are for their behavior (Wolfgang, 1996). Canter's discipline model (CDM), also known as Canter's approach, focuses on interaction and adheres to rules and their consequences based on reward and punishment mechanism (Canter \& Canter 2001). Malmgren, et al. (2005) advocate that children with undesired behavior should be treated with patience in discipline-inducing settings overseen by teachers (Canter \& Canter 2001).

Glasser's model of discipline (GMD), also known as reality therapy, argues that children should be encouraged to take responsibility for meeting their everyday life needs (Glasser, 1998). Teachers who adopt the GMD are more likely to help students establish causality between their behavior and its consequences and make the right choices (Irvine, 2015). Behavioral change theory (BCT) is another approach that focuses on behavior modification through the repetition of rewarded behavior under a controlled environment. The basic assumption of the BCT is that behavior can 
be changed by changing the conditioned stimuli following that behavior (Ozmon \& Craver 2008). Gordon's teacher effectiveness training (TET) model looks into how teachers can interact better with students (Talvio et al., 2013). TET advocates that undesired behavior can be reduced through open communication (Gordon, 2003).

Dreikurs' model of social discipline (MSD), also known as the logical consequences model, ensures that children understand that they are responsible for their behavior (Dreikurs, Grunwald, \& Pepper 1998). The MSD focuses on self-discipline, evaluation, and decision-making, and learning by doing. When faced with undesired behavior, teachers with the MSD can use the Ripple effect to prevent similar behavior in the future (Malmgren, Trezek, \& Paul 2005). Kounin's Discipline Model (KDM) takes individual needs into account to integrate activities through appropriate transitions. The KDM suggests that teachers should be tolerant and assume a holistic approach to factors that promote student engagement and reduce the prevalence of behavioral problems (Harlan \& Rowland 2002). These models are based on teacher-student interaction and need a safe, flexible, and supportive learning environment that meets student needs and promotes teacher-student interaction (Banks, 2014). Classroom management models facilitate learning, engagement, collaboration, and interaction more than conventional models (Oliver \& Reschly, 2007; Hamre et al., 2012; Türk, Kartal, Karademir \& Öcal, 2019).

\section{Teacher preparation programs}

Preschool teachers should encourage children to share their feelings and thoughts and develop social and cognitive skills and desired behavior (Oliver \& Reschly, 2007). However, it depends on how equipped and experienced they are. Those who do well in undergraduate studies are more likely to achieve that goal (Cains \& Brown, 1996). However, having a bachelor's degree does not guarantee high professional performance (Early et al., 2007; Emmer \& Stough, 2001). Preschool teachers should also interact with students positively and reassuringly (Bromfield, 2006) and organize classroom environments to encourage them to replace undesired behavior with the desired one. They should also be able to use activities to help preschoolers adopt desired behavior that will engage them in learning (Oliver \& Reschly, 2007; Early et al., 2007). Levine (2006) argues that preschool teachers who can do that are likely to have better classroom management. However, it is challenging for them to adhere to rules and carry out effective educational activities at the same time. Their ability to have effective classroom management depends on the knowledge and experience they acquire as undergraduates (O’Neill \& Stephenson, 2012). Preschool teachers are trained in classroom management. The purpose of vocational courses is to provide them with an 
opportunity to develop planning, organization, and management skills (Oliver \& Reschly, 2007). Teacher training programs differ significantly, but one question remains: Does theoretical teaching knowledge have the expected effect in practice? (Dicke et al., 2015). This raises another question: What content should the classroom management course (CMC) (an undergraduate vocational course) have, and how should it be put into practice to equip preschool teachers with relevant knowledge and skills? This study sought answers to this question.

Instructors of preservice teachers should know about their students' developmental characteristics and adopt educational approaches that best fit their needs. The right approaches allow instructors to evaluate all factors affecting the classroom atmosphere and identify positive and negative characteristics (Oliver \& Reschly, 2007). After choosing the right approach, the instructor should put the CMC into practice. Applied education programs help both instructors and preservice teachers develop professional skills (Weber et al., 2018). Preservice teachers need a sound theory and model to put classroom management decisions into practice (Dicke et al., 2015). However, instructors generally choose to use pseudo-models (Bromfield, 2006; Reupert \& Woodcock, 2010), and therefore, differ in the way they put CMCs into practice. Preservice teachers should know what model to use and in what context to use it (O’Neill \& Stephenson, 2012) and combine theoretical knowledge with practice. Only then can CMCs boost preservice teachers' confidence in choosing the right models.

\section{Research on classroom management models and preservice teacher education}

Preservice teachers have classroom management issues relating to undergraduate education (Bromfield, 2006; Öztürk, Gangal \& Ergişi, 2014; Reupert \& Woodcock, 2010; Swabey, Castleton \& Penney, 2010; Balli, 2011; Martin, 2004). However, earlier studies have focused little on the relationship between preservice education, behavior problems, and models (O’Neill \& Stephenson, 2012). Some researchers argue that current undergraduate education methods are too theoretical to meet all parties' needs. Therefore, they make suggestions to facilitate pre-service teachers' transition to professional life (Boe, Shin, \& Cook, 2007; Atici, 2007; Maskan, 2007). CMCs with activities enhance engagement, observation, and discussion and help students develop classroom management skills (Hamre et al., 2012; Wolff, Jarodzka \& Boshuizen, 2017). Moreover, conventional methods that focus on cognitive - but not on soft (noncognitive) skills - should be abandoned altogether (Bromfield, 2006; Shawer, 2017). Undergraduate courses should keep pace with the times and integrate video and 3D technology for interactive and applied learning (Straub, Dieker, Hynes \& Hughes, 2014; Cho, Mansfield \& Claughton, 2020; Weber et al., 2018). 
Behavior management is understudied in Turkey. One of them associates preschool teachers' classroom management skills with behavioral management models (Şahin-Sak et al., 2018), while a few others address classroom rules (Akar et al., 2010; Zembat, Tunçeli \& Akşin Yavuz, 2017). However, only a handful of them focuses on classroom management skills in preschool education (Akgün, Yarar \& Dinçer 2011; Bay, 2020; Toran \& Gençgel Akkus, 2016) and preservice teachers' beliefs, attitudes, and self-efficacy related to classroom management skills and models (Güleç, Bağçeli \& Onur 2008; Öztürk et al., 2014; Sak, 2015). Given the effects of discipline models on classroom activities, it is of paramount importance to determine why preservice teachers choose some classroom management models over others. However, only a limited number of studies address different models of discipline. For example, O’Neill and Stephenson (2012) looked into the effect of 22 classroom management models (including Gordon, Canter, and Glasser) on preservice teachers' perceptions and preferences (O’Neill \& Stephenson, 2012). Balli (2011) focused on behaviors based on Glasser models and examined preservice teachers' episodic memories of classroom management. Therefore, we believe that the results will help students develop desired behavior and classroom management skills and adopt new models.

\section{Research Objective}

Undergraduate CMCs should be of high-quality so that preschool teachers can provide good education. This study aimed to determine the variables affecting preservice preschool teachers' models and strategies to cope with undesired behavior in class. We believe that determining why preservice teachers prefer some models over others will help us (a) understand the relationship between those models and undergraduate CMCs, (b) figure out how to teach CMCs and to whom to teach them, and (c) find solutions to problems. Therefore, the study sought answers to the following questions:

1. Do gender, marital status, having taken the CMC before (course experience), and having a bachelor's degree (bachelor's degree status) affect preservice preschool teachers' choice of models to cope with undesired behaviors?

2. Do the areas of expertise of instructors teaching CMCs (instructors' areas of expertise) and the time since the foundation of the university (university experience) affect preservice preschool teachers' choice of models to cope with undesired behaviors? 


\section{METHOD}

\section{Research Model}

This correlational study determined the strength and direction of the relationship between variables without interfering with them (Fraenkel, Wallen \& Hyun, 2012). Therefore, the study looked into the effect of change in one variable on another.

\section{Participants}

The sample consisted of 1333 students from the preschool teaching department of seven Turkish universities. Ten students were excluded because they failed to complete the scales. Therefore, the final sample consisted of 1323 students (289 men; 1034 women). Participation was voluntary. The mean age was 22.11 years. First- and second-year students $(566 ; 42.8 \%)$ had not taken the CMC yet, while third- and fourth-year students $(757 ; 57.2 \%)$ had taken it before.

\section{Measures}

\section{Classroom Management Strategy Determination Scale (CMSDS)}

CMSDS is a 30-item measure developed by Keleş (2015). The CMSDS consists of six factors accounting for $49.89 \%$ of the total variance. The item factor loadings range from 0.32 to 0.76 . The subscales of the CMSDS are GMD (Items 1, 3, 6, 8, 14, 20, 23, 26, and 29), CDM (Items 2, 4, 7, 11, 13, 15, 25, and 27), TET (Items 5, 10, and 16), KDM (Items 17, 19, and 28), MSD (Items 9, 21, and 24), and BCT (Items, 12, 18, 22, and 30). Higher scores indicate a better ability to choose the right model. The CMSDS has a Cronbach's alpha of 0.71 (Keleş, 2015; Crocker \& Algina, 2006), which was 0.78 in this study, indicating acceptable internal consistency.

\section{Data collection methods}

The CMSDS was prepared on Google Forms. An electronic link was sent to all participants. Data were collected from seven universities because (1) they have different characteristics, and (2) the $\mathrm{CMC}$ in preschool teaching programs is taught by instructors from different areas of expertise.

\section{Data analysis}

First, Cronbach's alpha was calculated, and then, a correlation matrix was constructed using the mean and standard deviations of the subscales. Data were analyzed using JASP 0.10 .2 and Jamovi 1.2.0. 
The sample was nested (university-student), and therefore, scores were interdependent and affected by students and/or universities (Goldstein, 2011; Hox, 2010). For example, the students from a university may have similar characteristics that differentiate them from those of other universities (Finch, Bolin, \& Kelley, 2014; Osborne, 2000). Therefore, when collecting data from students from different universities, student- and university-level variables should be considered using the Hierarchical Linear Modeling (HLM) technique (Raudenbush \& Bryk, 2002; Wang, Xie, \& Fisher, 2012). This suggests that student and university levels should be included in the model separately or together (Hox, 2010) to analyze the hierarchical structure of the data (Goldstein, 2011). This study employed a two-level HLM method to determine the relationship between the models (CDM, GMD, BCT, TET, MSD, and KDM). Different models with two levels of independent (predictor) variables (Level 1: student and Level 2: university) were generated. Data were analyzed using HLM 7 (Raudenbush \& Bryk, 2002) at a significance level of 0.05.

On level 1, the scores of the models used by all participants were estimated as a function of gender $(1=$ female, $0=$ male $)$, marital status $(1=$ married, $0=$ single $)$, bachelor's degree status $(0=$ no, 1 $=$ yes $)$, and course experience $(0=$ not having taken the course, $1=$ having taken the course $)$. On level 2 , the scores of the models used by participants from different universities were estimated as a function of instructors' areas of expertise $(1=$ preschool education, $0=$ others $)$ and university experience. Sample size, variance homogeneity, error independence, and normality assumptions for errors were tested for Levels 1 and 2 before analysis (Raudenbush \& Bryk, 2002). The results satisfied the criteria, and therefore, HLM analysis was performed.

For modeling, One-Way ANOVA with Random Effects (Model 1), also known as the fully unconditional model, was used to determine whether scale scores differed across universities (Raudenbush \& Bryk, 2002). In Model 1, differences in the model scores (dependent variable) across the two levels were determined without including any independent variables. The following equations were generated for Model 1:

Model 1 (student level): Strategy $y_{i j}=\beta_{0 j}+r_{i j}, \operatorname{Var}\left(r_{i j}\right)=\sigma^{2}=$ within-group variance in strategy score.

where $S_{\text {trategy }} y_{j}$ is the model score of student $i$ from university $j, \beta_{0 j}$ is the mean model score of university $j$, and $r_{i j}$ is the error variance of student $i$ from university $j$. 
Model 2 (university level): $\beta_{0 j}=\gamma_{00}+u_{0 j}, \operatorname{Var}\left(u_{0 j}\right)=\tau=$ between group variance in strategy score.

where $\gamma_{00}$ is the mean of the model scores of $j$-number universities, $u_{0 j}$ is the random effect of university j, and $u_{0 j} \rightarrow 0$ indicates little variation across universities.

Mixed model: Strategy $y_{i j}=\gamma_{00}+u_{0 j}+r_{i j}$ (within-group model) shows the relationship between participants, while Model 2 (inter-group model) shows how that relationship varies across universities. The mixed model examines the variables of both participants' and universities' characteristics (Raudenbush \& Bryk, 2002). This allows us to use ANOVA to determine how much of the within-group variance is caused by the between-group variance.

If One-Way ANOVA with Random Effects shows that there is a difference in a dependent variable across universities (Woltman, Feldstain, MacKay \& Rocchi, 2012), then Means-as-Outcomes (Model 2) can be used to test the relationship between Level-2 independent (predictor) variables and students' scores (Raudenbush \& Bryk, 2002). The following equation shows the mixed model with only Level-2 predictor variables in Model 1:

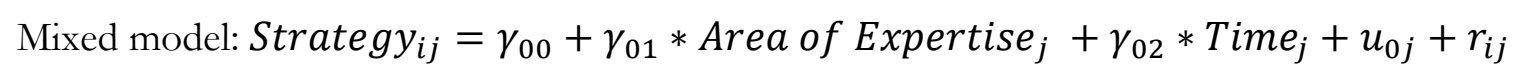

Only Level-2 predictor variables were included in the model to explain the difference between mean scores across universities. Afterwards, only Level-1 predictor variables were included in the model using Random-Coefficients (Model3) to determine the relationship between students' characteristics and scores (Raudenbush \& Bryk, 2002). The following equation shows the mixed model with only Level-1 predictor variables in Model 1:

Mixed model: $\quad$ Strategy $_{i j}=\gamma_{00}+\gamma_{10} *$ Gender $_{i j}+\gamma_{20} *$ Marital Status $_{i j}+\gamma_{30} *$ ${\text { Bachelor's } \text { Degree }_{i j}+\gamma_{40} * \text { Course Experience }}_{i j}+u_{0 j}+u_{1 j} *$ Gender $_{i j}+u_{2 j} *$

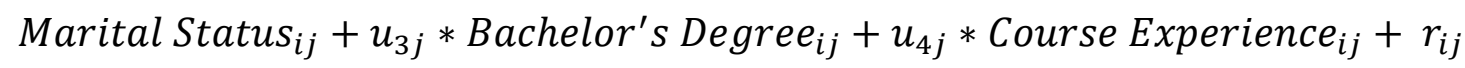

Only Level-1 predictor variables were included in the model to account for the difference in mean model scores across participants. Lastly, the predictor variables that were significant on Levels 1 and 2 were included in the model to obtain the full model, that is, the Intercepts-and Slopes-asOutcome Model (Model 4) (Raudenbush \& Bryk, 2002). The following equation shows the fully conditional mixed model with both Level-1 and Level-2 significant predictor variables in the unconditional model: 


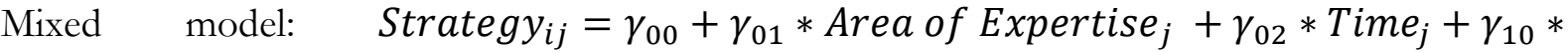

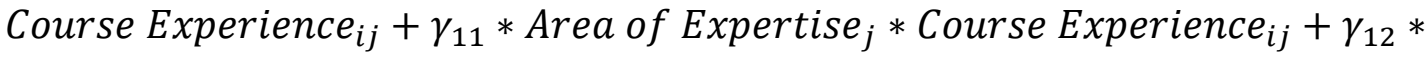

Time $_{j} *$ Course Experience $_{i j}+u_{0 j}+u_{1 j} *$ Course Experience Exj $_{i j}+r_{i j}$

The model was used to determine Level-1 variables affecting Level- 2 variables and the interaction between them. Equations developed for different models were used to calculate the effect size of independent variables on a dependent variable in practice (Woltman, Feldstain, MacKay \& Rocchi, 2012; Von Secker \& Lissitz, 1999; Sullivan \& Feinn, 2012). The criteria proposed by Raudenbush and Bryk (2002) were used to determine the reliability of the estimates.

\section{RESULTS}

\section{Descriptive Statistics and Correlations}

Table 1. Correlation matrix and descriptive statistics

\begin{tabular}{|c|c|c|c|c|c|c|c|c|c|c|c|c|c|c|}
\hline \multirow[b]{2}{*}{ Measures } & \multirow[b]{2}{*}{$\mathrm{M}$} & \multirow[b]{2}{*}{ SD } & \multicolumn{12}{|c|}{ Correlation } \\
\hline & & & 1 & 2 & 3 & 4 & 5 & 6 & 7 & 8 & 9 & 10 & 11 & 12 \\
\hline \multicolumn{15}{|l|}{ Student level } \\
\hline 1. Gender & & & & & & & .03 & .03 & $.06^{*}$ & .00 & $\begin{array}{l}- \\
.07 * *\end{array}$ & $.09 * *$ & -.05 & \\
\hline 2. Marital Status & & & & & & & $.12^{* *}$ & $.11 * *$ & $.12^{* *}$ & -.04 & .02 & .03 & $.08^{* *}$ & \\
\hline 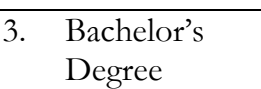 & & & & & & & $.07 * *$ & $.09 * *$ & $.06^{*}$ & -.04 & -.05 & .00 & $.13^{* *}$ & \\
\hline $\begin{array}{ll}4 . & \text { Course } \\
& \text { Experience } \\
\end{array}$ & & & & & & & $.34 * *$ & $.25^{* *}$ & $.20 * *$ & $.09 * *$ & -.02 & $.15^{* *}$ & $.32 * *$ & \\
\hline $\begin{array}{ll}5 . & \text { Model Total } \\
\text { Score }\end{array}$ & 106.89 & 8.53 & & & & & & $.64^{* *}$ & $.73^{* *}$ & $.43^{* *}$ & $.36^{* *}$ & $.39 * *$ & $.40^{* *}$ & \\
\hline 6. $\quad$ CDM & 27.82 & 3.45 & & & & & & & $.45^{* *}$ & -.02 & -.00 & $.12^{* *}$ & .03 & \\
\hline 7. GMD & 36.51 & 3.90 & & & & & & & & $.08^{* *}$ & $.11 * *$ & $.14^{* *}$ & .03 & \\
\hline 8. $\quad$ BCT & 11.94 & 2.44 & & & & & & & & & $.18^{* *}$ & .04 & $.25^{* *}$ & \\
\hline 9. TET & 11.04 & 2.03 & & & & & & & & & & .03 & $.06^{*}$ & \\
\hline 10. MSD & 9.87 & 2.05 & & & & & & & & & & & $.08^{* *}$ & \\
\hline 11. KDM & 9.74 & 2.28 & & & & & & & & & & & & \\
\hline \multicolumn{15}{|l|}{ University Level } \\
\hline $\begin{array}{l}\text { 12. Area of } \\
\text { expertise }\end{array}$ & & & & & & & $.28^{* *}$ & $.25^{* *}$ & $.23^{* *}$ & $.06^{*}$ & .05 & $.13^{* *}$ & $.06^{*}$ & \\
\hline $\begin{array}{l}\text { 13. University } \\
\text { Experience } \\
\text { (Year) }\end{array}$ & 35.99 & 20.81 & & & & & $.22^{* *}$ & $.18^{* *}$ & $.14^{* *}$ & $.06^{*}$ & .04 & $.19 * *$ & .05 & $\begin{array}{l}- \\
.10^{* *}\end{array}$ \\
\hline
\end{tabular}

Table 1 shows the descriptive statistics and correlation matrix. University experience was positively correlated with participants' CDM, GMD, BCT, and MSD scores. Female participants had higher GMD and MSD scores and lower TET scores than male participants. Married participants had higher CDM, GMD, and KDM scores than single participants. 


\section{Erken Çocukluk Çalışmaları Dergisi}

Cilt 5 Say1 1 Nisan 2021 s.152-179
Journal of Early Childhood Studies

Volume 5 Issue 1 Nisan 2021 pp.152-179

Participants with a bachelor's degree had higher CDM, GMD, and KDM scores than those without a bachelor's degree. Participants who had taken the CMC before (third- and fourth-year students) had higher CDM, GMD, BCT, MSD, and KDM scores than those who had not yet taken it (first- and second-year students). A two-level HLM analysis was used to determine the relationship between the variables and the student and university characteristics associated with participants' model scores.

\section{HLM results}

Table 2. HLM results for model total score

\begin{tabular}{|c|c|c|c|c|c|c|c|c|}
\hline \multirow[b]{2}{*}{ Fixed Effects } & \multicolumn{2}{|c|}{ Model1 } & \multicolumn{2}{|c|}{ Model2 } & \multicolumn{2}{|c|}{ Model3 } & \multicolumn{2}{|c|}{ Model4 } \\
\hline & $\beta$ & SE & $\beta$ & SE & $\beta$ & SE & $\beta$ & SE \\
\hline Intercept & $107.777 * * *$ & 1.458 & $107.784 * * *$ & 0.654 & $107.880^{* * *}$ & 1.583 & $107.883^{* * *}$ & 0.618 \\
\hline $\begin{array}{l}\text { Student Level } \\
\text { Gender }\end{array}$ & & & & & 0.022 & 0.419 & & \\
\hline Marital Status & & & & & 0.802 & 0.538 & & \\
\hline Bachelor's Degree & & & & & 0.716 & 0.637 & & \\
\hline Course Experience & & & & & $6.798^{* *}$ & 1.741 & $\begin{array}{c}6.693^{*} \\
0.068\end{array}$ & $\begin{array}{l}2.251 \\
0.070\end{array}$ \\
\hline University Level & & & & & & & & \\
\hline Area of expertise & & & $5.683^{*}$ & 1.361 & & & $6.690^{* *}$ & 1.274 \\
\hline University Experience (Year) & & & $0.116^{*}$ & 0.041 & & & $0.118^{*}$ & 0.040 \\
\hline Random Effects & $\mathrm{VC}$ & SD & $\mathrm{VC}$ & SD & VC & SD & VC & SD \\
\hline Student Level Variance $\left(\sigma^{2}\right)$ & 59.939 & 7.742 & 59.936 & 7.741 & 46.530 & 6.821 & 46.769 & 6.839 \\
\hline University Level Variance $\left(\tau_{00}\right)$ & 17.008 & 4.124 & 4.873 & 2.207 & 20.180 & 4.492 & 4.292 & 2.072 \\
\hline Reliability Estimate & & & 0. & & & & & \\
\hline
\end{tabular}

*** $\mathrm{p}<.001 ; * * \mathrm{p}<.01 ; * \mathrm{p}<.05 ; \mathrm{VC}$ : Variance Component 
The overall mean Model 1 (null) score was about 107.777, within-university variance $\left(\sigma^{2}\right)$ 59.939, and between-university variance $\left(\tau_{00}\right) 17.008$ (Table 2 ). The between-university variance showed that participants' total scores differed across universities $\left(\chi^{2}(6)=307.068, \mathrm{p}<.001\right)$. The total variance was divided into two levels to determine any significant difference in model scores across universities. The intraclass correlation coefficient showed that the variance in universities' scores accounted for about $22 \%$ of the total variance in participants' scores $(17.008 /(17.008+59.939))$. The results showed a statistically significant difference in participants' scores across universities, suggesting HLM for analysis.

Model 2 results showed that university experience $\left(\gamma_{02}=0.116, \mathrm{p}<.05\right)$ and instructors' areas of expertise $\left(\gamma_{01}=5.683, \mathrm{p}<.05\right)$ (Level-2 predictor variables) significantly predicted students' scores $\left(\chi^{2}(4)=72.039, \mathrm{p}<.001\right)$. The Level-2 predictor variables accounted for $71 \%[(17.008-4.873) /$ 17.008] of the total variance in universities' mean scores. The effect size was the gamma coefficients in the model divided by ANOVA between-university standard deviation (Von Secker \& Lissitz, 1999). The effect size showed that the students from universities with expert instructors had higher scores $(5.683 / \sqrt{17.008})(\mathrm{SD}=1.38)$ than those from universities without expert instructors. The students from experienced universities also had higher scores $(0.116 / \sqrt{17.008})(\mathrm{SD}=0.03)$ than those from inexperienced ones.

Model 3 included Level-1 predictor variables affecting students' scores. The results showed a change in the relationship between course experience and students' scores across universities $\left(\chi^{2}(6)\right.$ $=117.901, \mathrm{p}<.001)$ and students' scores from the same universities $\left(\gamma_{40}=6.798, \mathrm{p}<.01\right)$. These results indicated that students with course experience had a higher score by 6.798. There was, however, no relationship between students' scores and their gender $\left(\gamma_{10}=0.022, \mathrm{p}>.05\right)$, marital status $\left(\gamma_{20}=0.802, \mathrm{p}>.05\right)$, and bachelor's degree status $\left(\gamma_{30}=0.716, \mathrm{p}>.05\right)$. Course experience accounted for $22 \%$ [(59.939-46.530) / 59.939] of the total variance in students' scores. The effect size was the gamma coefficients in the model divided by ANOVA within-university standard deviation (Von Secker \& Lissitz, 1999). The effect size showed that third- and fourth-year students had higher scores $(6.798 / \sqrt{59.939})(S D=0.87)$ than first- and second-year students.

Model 4 included Level-1 and Level-2 predictor variables affecting students' scores. 


\section{Erken Çocukluk Çalışmaları Dergisi}

Cilt 5 Say1 1 Nisan 2021 s.152-179
Journal of Early Childhood Studies

Volume 5 Issue 1 Nisan 2021 pp.152-179

With the variable of "university experience" constant, the presence of expert instructors had a positive effect on universities' mean scores $\left(\gamma_{01}=6.690\right.$, $\mathrm{p}<.01)$. With the variable of “instructors' areas of expertise” constant, universities with more experience had higher mean scores $\left(\gamma_{02}=0.118, \mathrm{p}<.05\right)$.

Universities with expert instructors were likely to have higher scores than those without expert instructors $\left(\gamma_{11}=6.693\right.$, p<.05). Experienced universities had relatively higher scores than new universities; however, cross-level interaction with course experience was statistically insignificant $\left(\gamma_{12}=0.068\right.$, p >.05). In other words, university experience did not account for the between-university variance in course experience. In conclusion, analysis estimates had high reliability. Moreover, students who had taken the CMC (Level 1) from preschool experts (Level 2) had higher CMSDS scores. Table 3 shows the analysis results regarding the GMD and CDM subscales.

Table 3. HLM results for GMD and CDM subscales

\begin{tabular}{|c|c|c|c|c|c|c|c|c|}
\hline \multirow[b]{3}{*}{ Fixed Effects } & \multirow[b]{2}{*}{ Model1 } & \multicolumn{2}{|c|}{ GMD } & \multirow[b]{2}{*}{ Model4 } & \multirow[b]{2}{*}{ Model1 } & \multicolumn{2}{|c|}{ CDM } & \multirow[b]{2}{*}{ Model4 } \\
\hline & & Model2 & Model3 & & & Model2 & Model3 & \\
\hline & $\beta(\mathrm{SE})$ & $\beta(\mathrm{SE})$ & $\beta(\mathrm{SE})$ & $\beta(\mathrm{SE})$ & $\beta(\mathrm{SE})$ & $\beta(\mathrm{SE})$ & $\beta(\mathrm{SE})$ & $\beta(\mathrm{SE})$ \\
\hline Intercept & $36.842(0.496)^{* * *}$ & $36.840(0.215)^{* * *}$ & $36.854(0.566)^{* * *}$ & $36.834(0.198)^{* * *}$ & $28.148(0.501)^{* * *}$ & $28.149(0.216)^{* * *}$ & $28.216(0.552)^{* * *}$ & $28.196(0.266)^{* * *}$ \\
\hline Student Level & & & & & & & & \\
\hline Gender & & & $0.305(0.288)$ & & & & $0.125(0.162)$ & \\
\hline Marital Status & & & $0.871(0.450)$ & & & & $0.151(0.367)$ & \\
\hline Bachelor's Degree & & & $0.186(0.365)$ & & & & $0.764(0.159) * *$ & $-0.899(0.611)$ \\
\hline Status & & & $0.180(0.205)$ & & & & $0.104(0.153)$ & $0.004(0.013)$ \\
\hline Course Experience & & & $1.879(0.580) *$ & $1.768(0.781)$ & & & $2.074(0.602) *$ & $2.294(1.044)$ \\
\hline & & & & $0.009(0.022)$ & & & & $0.014(0.027)$ \\
\hline University Level & & & & & & & & \\
\hline Area of expertise & & $2.077(0.421)^{* *}$ & & $2.398(0.390)^{* *}$ & & $2.032(0.432)^{* *}$ & & $2.386(0.539)^{*}$ \\
\hline Experience (Year) & & $0.034(0.012)^{*}$ & & $0.034(0.012)^{*}$ & & $0.038(0.013)^{*}$ & & $0.039(0.014)^{*}$ \\
\hline Random Effects & $\mathrm{VC}(\mathrm{SD})$ & $\mathrm{VC}(\mathrm{SD})$ & $\mathrm{VC}(\mathrm{SD})$ & $\mathrm{VC}(\mathrm{SD})$ & VC(SD) & $\mathrm{VC}(\mathrm{SD})$ & VC(SD) & $\mathrm{VC}(\mathrm{SD})$ \\
\hline $\begin{array}{l}\text { Student Level } \\
\text { Variance }\left(\sigma^{2}\right)\end{array}$ & $13.822(3.718)$ & $13.821(3.718)$ & $12.664(3.559)$ & $12.774(3.574)$ & $10.372(3.221)$ & $10.371(3.220)$ & $9.038(3.006)$ & $9.072(3.012)$ \\
\hline $\begin{array}{l}\text { University Level } \\
\text { Variance }\left(\tau_{00}\right)\end{array}$ & $1.927(1.388)$ & $0.488(0.699)$ & $2.163(1.471)$ & $0.396(0.629)$ & $1.991(1.411)$ & $0.511(0.714)$ & $2.434(1.560)$ & $0.440(0.663)$ \\
\hline Reliability Estimate & 0.959 & 0.856 & 0.961 & 0.834 & 0.970 & 0.892 & 0.975 & 0.885 \\
\hline
\end{tabular}

*** $\mathrm{p}<.001 ;{ }^{* *} \mathrm{p}<.01 ;{ }^{*} \mathrm{p}<.05 ; \mathrm{VC}:$ Variance Component" 
Students' GMD $\left(\chi^{2}(6)=152.794, \mathrm{p}<.001\right)$ and CDM $\left(\chi^{2}(6)=214.936, \mathrm{p}<.001\right)$ scores differed across universities (Table 3). This suggested that between-university variance accounted for about $12 \%[1.927 /(1.927+13.822)]$ and $16 \%[1.991 /(1.991+10.372)]$ of the total variance in students' GMD and CDM scores, respectively. University experience and instructors' areas of expertise also had a significant effect on students' mean GMD $\left(\chi^{2}(4)=31.453, \mathrm{p}<.001\right)$ and $\operatorname{CDM}\left(\chi^{2}(4)\right.$ $=44.859, \mathrm{p}<.001)$ scores. This suggested that instructors' areas of expertise $\left(\gamma_{01_{G D M}}=2.077, \mathrm{p}<.05\right.$; $\left.\gamma_{01_{C D M}}=2.032, \mathrm{p}<.05\right)$ and university experience $\left(\gamma_{02_{G D M}}=0.034, \mathrm{p}<.05 ; \gamma_{02}{ }_{C D M}=0.038, \mathrm{p}<.05\right)$ significantly predicted students' GMD and CDM scores. Level-2 predictor variables accounted for about 75\% [(1.927-0.488) / 1.927] and 74\% [(1.991-0.510) / 1.991] of the total variance in students' GMD and CDM scores, respectively. The effect size showed that students from universities with expert instructors had higher GMD $(2.077 / \sqrt{1.927})(\mathrm{SD}=1.49)$ and $\mathrm{CDM}(2.032 / \sqrt{1.991})$ $(\mathrm{SD}=2.86)$ scores than those from universities without expert instructors. Students from experienced universities also had higher $\operatorname{GMD}(0.034 / \sqrt{1.927})(\mathrm{SD}=0.02)$ and $\mathrm{CDM}$ $(0.038 / \sqrt{1.991})(\mathrm{SD}=0.05)$ scores than those from inexperienced ones.

In Model 3, the relationship between course experience and GMD scores differed across universities $\left(\chi^{2}(6)=41.094, \mathrm{p}<.001\right)$. There was a significant relationship between course experience and GMD scores $\left(\gamma_{40}=1.879, \mathrm{p}<.05\right)$. However, students' gender $\left(\gamma_{10}=0.305, \mathrm{p}>.05\right)$, marital status $\left(\gamma_{20}=0.871, \mathrm{p}>.05\right)$, and bachelor's degree status $\left(\gamma_{30}=0.186, \mathrm{p}>.05\right)$ had no effect on their GMD scores. Third- and fourth-year students had higher GMD scores $(1.879 / \sqrt{13.822})$ $(\mathrm{SD}=0.50)$ than first- and second-year students. Level-1 variables accounted for 8\% [(13.82212.664) / 13.822] of the total variance in students' GMD scores. We moved on to Model 4 because Models 2 and 3 had significant predictors. Model 3 analysis for CDM showed that course experience and CDM scores differed across universities $\left(\chi^{2}(6)=68.397, \mathrm{p}<.001\right)$. Moreover, there was a significant relationship between students' CDM scores and course experience $\left(\gamma_{40}=2.074\right.$, $\mathrm{p}<.05)$ and bachelor's degree status $\left(\gamma_{30}=0.764, \mathrm{p}<.05\right)$. However, gender $\left(\gamma_{10}=0.125, \mathrm{p}>.05\right)$ and marital status $\left(\gamma_{20}=0.151, \mathrm{p}>.05\right)$ had no effect on their CDM scores. Third- and fourth-year students had higher $\mathrm{CDM}$ scores $(2.074 / \sqrt{10.372})(\mathrm{SD}=0.64)$ than first- and second-year students. Students with a bachelor's degree had higher CDM scores $(0.764 / \sqrt{10.372})(\mathrm{SD}=0.23)$ than those without a bachelor's degree. Level-1 variables accounted for $12 \%$ [(10.3729.038)/10.372] of the total variance (effect size) in students' CDM scores. 


\section{Erken Çocukluk Çalışmaları Dergisi}

Cilt 5 Sayı 1 Nisan 2021 s.152-179
Journal of Early Childhood Studies

Volume 5 Issue 1 Nisan 2021 pp.152-179

Although it was quite low, we moved on to Model 4 because Models 2 and 3 had significant predictors. As for the GMD scores, the cross-level interaction across students who had or had not learned the CMC from preschool experts or instructors from different branches was statistically insignificant $\left(\gamma_{11}=1.768, \mathrm{p}>.05\right)$. Similarly, the cross-level interaction across students who had or had not taken the CMC at experienced or new universities was statistically insignificant $\left(\gamma_{-} 12=0.009, \mathrm{p}>.05\right)$. Level-1 variables accounted for only $8 \%$ of students' GMD scores, and therefore, the cross-level interaction was statistically insignificant. The estimates for the GMD had high reliability. This showed that the cross-level interaction of instructors' areas of expertise and university experience did not account for between-university variance in students' course experience.

Table 4. HLM results for BCT and TET subscales

\begin{tabular}{|c|c|c|c|c|c|c|c|c|}
\hline \multirow[b]{3}{*}{ Fixed Effects } & \multirow[b]{2}{*}{ Model1 } & \multicolumn{2}{|c|}{ BCT } & \multirow[b]{2}{*}{ Model4 } & \multirow[b]{2}{*}{ Model1 } & \multicolumn{2}{|c|}{ TET } & \multirow[b]{2}{*}{ Model4 } \\
\hline & & Model2 & Model3 & & & Model2 & Model3 & \\
\hline & $\beta(\mathrm{SE})$ & $\beta(\mathrm{SE})$ & $\beta(\mathrm{SE})$ & $\beta(\mathrm{SE})$ & $\beta(\mathrm{SE})$ & $\beta(\mathrm{SE})$ & $\beta(\mathrm{SE})$ & $\beta(\mathrm{SE})$ \\
\hline Intercept & $11.945(0.152)^{* * *}$ & $11.953(0.133)^{* * *}$ & $11.953(0.158)^{* * *}$ & $11.965(0.132)^{* * *}$ & $11.064(0.092)^{* * *}$ & $11.076(0.072)^{* * *}$ & $11.064(0.103)^{* * *}$ & $11.073(0.098)^{* * *}$ \\
\hline Student Level & & & & & & & & \\
\hline Gender & & & $-0.083(0.126)$ & & & & $-0.394(0.173)$ & \\
\hline Marital Status & & & $-0.279(0.306)$ & & & & $0.303(0.284)$ & \\
\hline Bachelor's & & & $-0.378(0.225)$ & & & & $-0.485(0.210)$ & \\
\hline $\begin{array}{l}\text { Degree Status } \\
\text { Course }\end{array}$ & & & & & & & & \\
\hline $\begin{array}{l}\text { Course } \\
\text { Experience }\end{array}$ & & & $0.541(0.228)$ & & & & $-0.019(0.211)$ & \\
\hline University Level & & & & & & & & \\
\hline Area of expertise & & $0.319(0.311)$ & & & & $0.281(0.156)$ & & \\
\hline $\begin{array}{l}\text { Experience } \\
\text { (Year) }\end{array}$ & & $0.008(0.005)$ & & & & $0.005(0.005)$ & & \\
\hline Random Effects & VC(SD) & VC(SD) & VC(SD) & VC(SD) & VC(SD) & VC(SD) & VC(SD) & $\mathrm{VC}(\mathrm{SD})$ \\
\hline $\begin{array}{l}\text { Student Level } \\
\text { Variance }\left(\sigma^{2}\right)\end{array}$ & $5.859(2.420)$ & $5.859(2.420)$ & $5.716(2.391)$ & $5.759(2.399)$ & $4.104(2.026)$ & $4.102(2.025)$ & $4.010(2.003)$ & $4.055(2.013)$ \\
\hline $\begin{array}{l}\text { University Level } \\
\text { Variance }\left(\tau_{00}\right)\end{array}$ & $0.156(0.394)$ & $0.167(0.409)$ & $0.176(0.420)$ & $0.167(0.408)$ & $0.045(0.213)$ & $0.043(0.208)$ & $0.052(0.227)$ & $0.044(0.209)$ \\
\hline $\begin{array}{l}\text { Reliability } \\
\text { Estimate }\end{array}$ & 0.818 & 0.828 & 0.819 & 0.824 & 0.657 & 0.646 & 0.660 & 0.645 \\
\hline
\end{tabular}

${ }^{* * *} \mathrm{p}<.001 ;{ }^{* *} \mathrm{p}<.01 ;{ }^{*} \mathrm{p}<.05 ;$ VC: Variance Component 
As for the cross-level interactions of Level 1 and Level 2 predictor variables in the CDM subscale; cross-level interaction between course experience and instructors' areas of expertise $\left(\gamma_{21}=2.294\right.$, $\mathrm{p}>.05)$ and university experience $\left(\gamma_{22}=0.014, \mathrm{p}>.05\right)$ was statistically insignificant. Moreover, cross-level interaction between bachelor's degree status and instructors' areas of expertise $\left(\gamma_{11}=\right.$ $0.899, \mathrm{p}>.05$ ) and university experience was statistically insignificant. Level-1 variables accounted for only $12 \%$ of the total variance in students' CDM scores, and therefore, the cross-level interaction was statistically insignificant. Overall, the estimates for the CDM had high reliability. The result showed that the between-university variance in course experience and bachelor's degree status was not related to the cross-level interaction of university experience and instructors' areas of expertise. Table 4 shows the analysis results regarding the BCT and TET subscales.

Students' BCT $\left(\chi^{2}(6)=30.880, \mathrm{p}<.001\right)$ and TET $\left(\chi^{2}(6)=17.223, \mathrm{p}<.01\right)$ scores varied across universities (Table 4). This suggested that between-university variance accounted for about 3\% $(0.155 /(0.155+5.859))$ and $1 \%(0.045 /(0.045+4.104))$ of the total variance in their BCT and TET scores, respectively. With the variables of "university experience" and “instructors' areas of expertise" constant, their BCT $\left(\chi^{2}(4)=20.656, \mathrm{p}<.001\right)$ and TET $\left(\chi^{2}(4)=12.302, \mathrm{p}<.05\right)$ scores significantly changed. However, instructors' areas of expertise $\left(\gamma_{01_{B C T}}=0.319, \mathrm{p}>.05\right.$; $\left.\gamma_{01_{T E T}}=0.281, \mathrm{p}>.05\right)$ and university experience $\left(\gamma_{02_{B C T}}=0.008, \mathrm{p}>.05 ; \gamma_{02_{T E T}}=0.005, \mathrm{p}>.05\right)$ did not significantly predict their BCT and TET scores. Therefore, including Level-2 predictor variables in the model did not result in an increase in their BCT and TET scores.

In Model 3, the relationship between students' course experience and BCT scores varied across universities $\left(\chi^{2}(6)=18.167, \mathrm{p}<.01\right)$. However, course experience $\left(\gamma_{40}=0.541, \mathrm{p}>.05\right)$, gender $\left(\gamma_{10}=-0.083, \mathrm{p}>.05\right)$, bachelor's degree status $\left(\gamma_{30}=-0.378, \mathrm{p}>.05\right)$, and marital status $\left(\gamma_{20}=-0.279\right.$, $\mathrm{p}>.05)$ had no significant effect on their BCT scores, with Level-1 variables accounting only $2 \%$ [(5.859-5.716) / 5.859] of the total variance. The estimates for BCT had high reliability. The variables in Models 2 and 3 were statistically insignificant, and therefore, none of the cross-level interactions of Level-1 and Level-2 variables was statistically significant. 


\section{Erken Çocukluk Çalışmaları Dergisi}

Cilt 5 Say1 1 Nisan 2021 s.152-179
Journal of Early Childhood Studies

Volume 5 Issue 1 Nisan 2021 pp.152-179

The relationship between course experience and TET scores also varied across universities $\left(\chi^{2}(6)=16.604, \mathrm{p}<.05\right)$. Course experience $\left(\gamma_{40}=-0.019\right.$, $\mathrm{p}>.05)$, gender $\left(\gamma_{10}=-0.394, \mathrm{p}>.05\right)$, bachelor's degree status $\left(\gamma_{30}=-0.485, \mathrm{p}>.05\right)$, and marital status $\left(\gamma_{20}=0.303\right.$, $\left.\mathrm{p}>.05\right)$ had no effect on students' TET scores, with Level-1 variables accounting for only 2\% [(4.104-4.010) / 4.104] of the total variance. The estimates for TET had moderate reliability. The variables in Models 2 and 3 were statistically insignificant, and therefore, none of the cross-level interactions of Level-1 and Level-2 variables was statistically significant. Table 5 shows the analysis results regarding the MSD and KDM subscales.

Table 5. HLM results for BCT and TET subscales

\begin{tabular}{|c|c|c|c|c|c|c|c|c|}
\hline \multirow[b]{3}{*}{ Fixed Effects } & \multirow[b]{2}{*}{ Model1 } & \multicolumn{2}{|c|}{ MSD } & \multirow[b]{2}{*}{ Model4 } & \multirow[b]{2}{*}{ Model1 } & \multicolumn{2}{|c|}{ KDM } & \multirow[b]{2}{*}{ Model4 } \\
\hline & & Model2 & Model3 & & & Model2 & Model3 & \\
\hline & $\beta(\mathrm{SE})$ & $\beta(\mathrm{SE})$ & $\beta(\mathrm{SE})$ & $\beta(\mathrm{SE})$ & $\beta(\mathrm{SE})$ & $\beta(\mathrm{SE})$ & $\beta(\mathrm{SE})$ & $\beta(\mathrm{SE})$ \\
\hline Intercept & $10.000(0.199)^{* * *}$ & $10.002(0.091)^{* * *}$ & $10.006(0.205)^{* * *}$ & $9.995(0.104)^{* * *}$ & $9.754(0.133)^{* * *}$ & $9.764(0.101)^{* * *}$ & $9.786(0.174)^{* * *}$ & $9.782(0.153)^{* * *}$ \\
\hline $\begin{array}{l}\text { Student Level } \\
\text { Gender }\end{array}$ & & & $0.420(0.118)^{*}$ & $\begin{array}{l}0.274(0.319) \\
0.009(0.007)\end{array}$ & & & $-0.293(0.150)$ & \\
\hline Marital Status & & & $-0.100(0.125)$ & & & & $-0.101(0.288)$ & \\
\hline Bachelor's degree Status & & & $0.065(0.129)$ & & & & $0.523(0.216)$ & \\
\hline Course experience & & & $0.696(0.217)^{*}$ & $\begin{array}{c}0.585(0.474) \\
-0.007(0.012)\end{array}$ & & & $1.567(0.324)^{* *}$ & $\begin{array}{c}0.795(0.261)^{*} \\
0.032(0.006)^{* *}\end{array}$ \\
\hline University Level & & & & & & & & \\
\hline Area of expertise & & $0.588(0.175)^{*}$ & & $0.714(0.211)^{*}$ & & $0.382(0.214)$ & & $0.579(0.312)$ \\
\hline Experience (Year) & & $0.020(0.004)^{* *}$ & & $0.020(0.005)^{*}$ & & $0.007(0.006)$ & & $0.007(0.008)$ \\
\hline Random Effects & $\mathrm{VC}(\mathrm{SD})$ & $\mathrm{VC}(\mathrm{SD})$ & $\mathrm{VC}(\mathrm{SD})$ & $\mathrm{VC}(\mathrm{SD})$ & $\mathrm{VC}(\mathrm{SD})$ & $\mathrm{VC}(\mathrm{SD})$ & $\mathrm{VC}(\mathrm{SD})$ & $\mathrm{VC}(\mathrm{SD})$ \\
\hline $\begin{array}{l}\text { Student Level Variance } \\
\left(\sigma^{2}\right)\end{array}$ & $3.950(1.988)$ & $3.951(1.988)$ & $3.750(1.937)$ & $3.755(1.938)$ & $5.138(2.266)$ & $5.137(2.266)$ & $4.410(2.100)$ & $4.456(2.110)$ \\
\hline $\begin{array}{l}\text { University Level Variance } \\
\left(\tau_{00}\right)\end{array}$ & $0.299(0.547)$ & $0.078(0.280)$ & $0.319(0.565)$ & $0.053(0.230)$ & $0.113(0.337)$ & $0.102(0.319)$ & $0.186(0.431)$ & $0.137(0.371)$ \\
\hline Reliability Estimate & 0.927 & 0.772 & 0.925 & 0.698 & 0.790 & 0.771 & 0.860 & 0.834 \\
\hline
\end{tabular}

*** $\mathrm{p}<.001 ;{ }^{* *} \mathrm{p}<.01 ;{ }^{*} \mathrm{p}<.05 ; \mathrm{VC}:$ Variance Component 
Students' $\operatorname{MSD}\left(\chi^{2}(6)=102.156, \mathrm{p}<.001\right)$ and $\operatorname{KDM}\left(\chi^{2}(6)=26.854, \mathrm{p}<.001\right)$ scores varied across universities. Between-university variance accounted for about 7\% (0.298 / (0.298+3.950)) and 2\% $(0.113 /(0.113+5.138))$ of the total variance in their MSD and KDM scores, respectively (Table $5)$.

With the variables of "university experience" and "instructors' areas of expertise" constant, students' $\operatorname{MSD}\left(\chi^{2}(4)=17.934, \mathrm{p}<.01\right)$ and $\operatorname{KDM}\left(\chi^{2}(4)=19.690, \mathrm{p}<.001\right)$ scores significantly changed. Instructors' areas of expertise $\left(\gamma_{01_{S D M}}=0.588, \mathrm{p}<.05 ; \gamma_{01_{K D M}}=0.382, \mathrm{p}>.05\right)$ and university experience $\left(\gamma_{02}{ }_{S D M} 2=0.020, \mathrm{p}<.05 ; \gamma_{02_{K D M}}=0.007, \mathrm{p}>.05\right)$ significantly predicted students' MSD scores, but not their KDM scores. Level-2 predictor variables accounted for about $73 \%$ [(0.298-0.078) / 0.298] of the total variance in their MSD scores. The effect size showed that students from universities with expert instructors had higher MSD scores $(0.588 / \sqrt{0.078})(\mathrm{SD}=$ 0.16) than those from universities without expert instructors. Students from experienced universities had higher MSD scores $(0.020 / \sqrt{0.078})(\mathrm{SD}=0.005)$ than those from new universities. Level-2 predictor variables did not significantly predict KDM scores; hence, no variance in students' KDM scores.

In Model 3, students' course experience and MSD scores varied across universities $\left(\chi^{2}(6)=27.128\right.$, $\mathrm{p}<.001)$. Course experience $\left(\gamma_{40}=0.696, \mathrm{p}<.05\right)$ and gender $\left(\gamma_{10}=0.420, \mathrm{p}<.05\right)$ also had a significant effect on their MSD scores. On the other hand, bachelor's degree status $\left(\gamma_{30}=0.065\right.$, $\mathrm{p}>.05)$ and marital status $\left(\gamma_{20}=-0.100, \mathrm{p}>.05\right)$ had no significant effect on their MSD scores. Thirdand fourth-year students had higher MSD scores $(0.696 / \sqrt{3.950})(\mathrm{SD}=0.35)$ than first- and second-year students. Female students had higher MSD scores $(0.420 / \sqrt{3.950})(\mathrm{SD}=0.21)$ than males. Level-1 variables accounted for only 5\% [(3.950-3.750) / 3.950] of the total variance in students' MSD scores, but we moved on to Model 4 because Model 2 and Model 3 had significant predictors.

Students' course experience and KDM scores varied across universities $\left(\chi^{2}(6)=44.836, \mathrm{p}<.001\right)$. There was also a significant relationship between course experience and KDM scores $\left(\gamma_{40}=1.567\right.$, $\mathrm{p}<.05)$. However, gender $\left(\gamma_{10}=-0.293, \mathrm{p}>.05\right)$, marital status $\left(\gamma_{20}=-0.101, \mathrm{p}>.05\right)$, and bachelor's degree status $\left(\gamma_{30}=0.523, \mathrm{p}>.05\right)$ had no effect on their KDM scores. Third- and fourth-year students had higher KDM scores $(1.567 / \sqrt{5.138})(\mathrm{SD}=0.69)$ than first- and second-year 
students. Level-1 variables accounted for $\% 14$ of the total variance in students' KDM scores. Course experience was statistically significant in Model 3, and therefore, Model 4 was generated to investigate cross-level interactions.

The estimates for the MSD had average or above-average reliability. The cross-level interactions of Level-1 and Level-2 predictor variables showed that the interaction between students' course experience and instructors' areas of expertise $\left(\gamma_{21}=0.585, \mathrm{p}>.05\right)$ and university experience $\left(\gamma_{22}\right.$ $=-0.007, \mathrm{p}>.05$ ) was statistically insignificant. Moreover, the cross-level interaction between gender and instructors' areas of expertise $\left(\gamma_{11}=0.274, \mathrm{p}>.05\right)$ and university experience $\left(\gamma_{12}=0.009\right.$, p>.05) was statistically insignificant. Level-1 variables accounted for only a low percentage of students' MSD scores, and therefore, the cross-level interaction was statistically insignificant. This result suggested that the between-university variance in course experience and gender had no interaction with university experience and instructors' areas of expertise.

According to Model 4 results for the KDM, universities with expert instructors had higher KDM scores than those without expert instructors $\left(\gamma_{11}=0.795, \mathrm{p}<.05\right)$. Experienced universities also had higher KDM scores than new universities, and its cross-level interaction with students' course experience was statistically significant $\left(\gamma_{12}=0.032, \mathrm{p}<.05\right)$. In other words, the between-university variance in the course experience was related to the university experience. The estimates for KDM had moderate and above moderate reliability. In conclusion, students who had learned the CMC (student level) from preschool experts at experienced universities (school level) had higher KDM scores.

\section{CONCLUSION and DISCUSSION}

This study determined student- and university-related characteristics causing differences in behavioral management model scores used by preservice preschool teachers from seven universities. Students' gender, marital status, bachelor's degree status, and course experience, as well as university experience, and instructors' areas of expertise were the student- and universityrelated characteristics in question. Students' total model score and mean GMD, CDM, and MSD scores significantly varied across universities, while their KDM, TET, and BCT scores varied across universities at a low level. HLM technique was used to determine the relationship between variables at different levels (preservice teacher -university). 


\section{Child-centered discipline models}

As for the total model score and the GMD and MSD scores, students who had taken the CMC from preschool experts at experienced universities were more likely to use the right models. Students who had taken the CMC from preschool experts had higher KDM scores. This result shows that preservice teachers who take the undergraduate CMC from experts are more likely to improve themselves. The quality of training received by preservice teachers depends on instructors' qualifications (Martin, 2004; Parpucu, Yıldırım-Polat \& Akman, 2018). Experienced universities have better infrastructure and resources and more expert instructors than new ones. Our results also show that preservice preschool teachers who have received education at experienced universities are better at choosing the right models to cope with the undesired behavior.

Four in ten students who think they have received high-quality undergraduate education have behavior management issues (Boe et al.,2007). Therefore, students trained by preschool experts are more likely to base their knowledge on observation, discussion, and practice (Bromfield, 2006). Preservice teachers who use the GMD and MSD models are more likely to collaborate with students to manage the class and encourage them to think about the relationship between their behavior and its consequences (Levine \& He, 2008; Balli, 2011).

Expert instructors should focus on practice rather than theory in CMCs to teach preservice teachers how to cope with undesired behavior and communicate effectively with students (Rathel, Drasgow \& Christle, 2008). Research suggests that instructors should involve preservice teachers in activities to increase their readiness for classroom management. For example, researchers focus on emotionally supportive classroom management education (Hu et al., 2016; Dicke et al., 2015), practice-oriented and verbal-nonverbal active supervision professional competence development for foresight (Weber et al., 2018; Wollf et al., 2017), experience-focused experience (Balli, 2011; Opdenakker \& Van Damme, 2006), and modern classrooms (Cho et al., 2014).

Dreikurs' model of social discipline (MSD) asserts that undesired behavior is more prevalent in children with unmet social needs. Teachers who adopt MSD are more likely to effectively communicate with students (Soheili et al., 2015). Female preservice teachers who have taken CMCs are better at choosing the right MSD models than male preservice teachers. This may be because women have more effective listening, comprehension, and verbal skills (tone of voice, eye contact, addressing by name, physical intimacy, etc.) than men (Aylor, 2003). However, numerous earlier studies have argued that male and female preservice teachers do not differ in the way they choose behavior management models (O’Neill \& Stephenson, 2012; Şahin-Sak et al., 2018) and interact 
with children (Brandes et al., 2015; Sak, Şahin Sak \& Yerlikaya 2015; Toran \& Gençgel Akkuş, 2016).

\section{Teacher-centered discipline models}

Many countries still use the CDM and BCT models, which focus on conventional teacher-child interaction and advocate for the preservation of assertive but positive attitude while giving control to teachers [see, for example, Balli, 2011 (USA); O’Neill \& Stephenson, 2012 (Australia); Akar et al., 2010 (Turkey)]. Another interesting result was that students with a bachelor's degree had higher CDM scores. It would not be wrong to assume that it is because the Turkish education system is still based on reward and punishment. Preservice teachers feel most confident when they use praise, encouragement, and reward (O’Neill \& Stephenson, 2012), which are also the main components of the CDM and BCT models. Therefore, our results confirm that preschool teachers generally collaborate with students to lay down rules. Our participants had had different teachers with different classroom management skills for ten years (from Pre-K to high school) before enrolling in teacher training programs. Therefore, their positive and negative experiences with their instructors may have influenced the way they completed the scale (Balli, 2011).

Preschool teachers in many countries use rewards and punishments to manage their classrooms (Balli, 2011; Ozmon \& Craver, 2008; Şahin-Sak et al., 2018) because preschool education programs instruct them to condition students for desired behavior and rules (Dicke et al., 2015; MoNe, 2013; Oliver \& Reschly, 2007). Teachers use CDM and BCT to regulate their students' behavior, engage them in educational activities, and reduce their destructive behavior through rewards and punishments (Wollf et al., 2017). However, they forget that CDM and BCT are teacher-centered conventional models (Pianta, 2006). Teachers use BCT to retain control to enforce their own classroom rules and carry out procedures without disruption (Ozmon \& Craver, 2008). Teachers of young children (3-4 years of age) generally use those types of models to attract students' attention, enforce classroom rules, and replace undesired behavior with desired behavior (Dinçer \& Akgün 2015; Şahin-Sak et al., 2018). Our participants completed the scale without any direct focus on a particular age group and chose the BCT model over others, which may be because it is an easy-to-use model based on reward and punishment (O’Neill \& Stephenson, 2012).

Course experience helped our participants choose the right models (GMD, CDM, MSD, and KDM). This suggests that most university instructors address the models in teacher training programs and books (O’Neill \& Stephenson, 2012). However, taking CMCs alone does not guarantee the development of teaching skills (Parpucu et al., 2018). High-quality education also 
requires interaction and collaboration with students (Brophy, 2006). Therefore, preservice teachers should learn $\mathrm{CMCs}$ and receive feedback from expert instructors to be able to learn teaching methods and develop skills (Early et al., 2017; Hamre et al., 2012; Hu et al., 2016; Pianta, Mashburn et al., 2008).

Another result was that university experience and taking CMCs from experts did not affect participants' BCT, KDM, and TET scores. This shows that students from universities with different demographic characteristics choose those three models to condition students for desired behavior. This is not a surprising result, given the fact that preservice teachers generally adopt models with reactionary and preventive strategies (Reupert \& Woodcock, 2010). Participants' perceptions of the six behavior management models were affected by different variables. They had similar perceptions for Glasser's discipline model, Dreikurs' social discipline model, and Canter's assertive discipline model, which involve, or they think they involve, child-centered strategies. Moreover, participants who had taken the CMC were more likely to choose the right classroom management models. They had higher GMD, MSD, and KDM (child-centered models) and CDM scores (a model used to teach small children about rules). This result indicates that preservice teachers should take undergraduate CMCs from experts. Gender was an important factor in Dreikurs' social discipline model. Participants were able to distinguish between child-centered approaches and conventional teacher-centered models, which is a promising result.

\section{Limitations and future directions}

Universities in Turkey have similar curricula for preservice teachers of early childhood education. Therefore, preschool teachers' performance in professional life depends on who teaches them and how they teach. To be more precise, this study highlights once again that expert instructors should deliver CMCs. The results can help curriculum developers improve the quality of CMCs offered by all universities. The research is limited to seven universities in Turkey. Therefore, future studies should focus on preservice teachers from different countries.

Universities should make sure that expert instructors deliver CMCs. Preservice teachers should be offered more CMCs that focus on practice because they need a robust theory and model to put their decisions concerning classroom management into practice. Classrooms should be equipped with technological infrastructure (simulation, video, augmented reality, and 3D). Teachers who have problems with classroom management should be provided with comparative in-service training. School-university cooperation should be encouraged. Future qualitative and/or longitudinal research with smaller sample sizes should be performed to confirm the results. 


\section{REFERENCES}

Akar, H., F. Tantekin-Erden, D. Tor, \& İ. T. Şahin. 2010. Öğretmenlerin sınıf yönetimi yaklaşımları ve deneyimlerinin incelenmesi [Study on Teachers' Classroom Management Approaches and Experiences]. İlkögretim Online [Elementary Education Online] 9 (2): 792-806.

Akgün, E., M. Yarar, \& Ö. Dinçer. 2011. Okul Öncesi öğretmenlerinin sınıf içi etkinliklerde kullandıkları sınıf yönetimi stratejilerinin incelenmesi [The Evaluation of Classroom Management Strategies of Preschool Teachers in Classroom Activities]. Pegem Eğitim ve Ögretim Dergisi [Pegem Journal of Education and Instruction] 1 (3): 1-9.

Atici, M. (2007). A small-scale study on student teachers' perceptions of classroom management and methods for dealing with misbehaviour. Emotional and Behavioural Difficulties, 12(1), 1527.

Aylor, B. 2003. The Impact of sex, gender, and cognitive complexity on the perceived importance of teacher communication skills. Communication Studies 54 (4): 496-509.

Baker, P. H. (2005). Managing student behavior: How ready are teachers to meet the challenge?. American secondary education, 51-64.

Balli, S. J. (2011). Pre-service teachers' episodic memories of classroom management. Teaching and Teacher Education, 27(2), 245-251.

Banks, T. (2014). Creating positive learning environments: Antecedent strategies for managing the classroom environment \& student behavior. Creative Education, 5, 519-524.

Bay, D. N. (2020). Investigation of the relationship between self-efficacy beliefs and classroom management skills of pre-school teachers. International Electronic Journal of Elementary Education, 12(4), 335-348.

Beaman, R., Wheldall, K., \& Kemp, C. (2007). Recent research on troublesome classroom behaviour: A review. Australasian Journal of Special Education, 31(1), 45-60.

Bear, G. (2014). Preventive and classroom-based strategies. In E. T. Emmer \& E. J. Sabornie (Eds.), Handbook of classroom management (pp. 15-39). New York, NY: Routledge.

Blazar, D., \& Kraft, M. A. (2017). Teacher and teaching effects on students' attitudes and behaviors. Educational evaluation and policy analysis, 39(1), 146-170.

Boe, E. E., Shin, S., \& Cook, L. H. (2007). Does teacher preparation matter for beginning teachers in either special or general education? The Journal of Special Education, 41, 158-170.

Brandes, H., M. Andrä, W. Röseler, \& P. Schneider-Andrich, 2015. Does gender make a difference? results from the german 'tandem study' on the pedagogical activity of female and male ECE workers. European Early Childhood Education Research Journal 23 (3): 315-327.

Bromfield, C. (2006). PGCE secondary trainee teachers \& effective behaviour management: An evaluation and commentary. Support for Learning, 21(4), 188-193. 
Brophy, J. (2006). History of research on classroom management. In C. M. Evertson, \& C. S. Weinstein (Eds.), Handbook of classroom management, research, practice and contemporary issues (pp. 17-43). Mahwah, NJ: Erlbaum.

Buyse, E., Verschueren, K., Doumen, S., Van Damme, J., \& Maes, F. (2008). Classroom problem behavior and teacher-child relationships in kindergarten: The moderating role of classroom climate. Journal of School Psychology, 46(4), 367-391.

Cains, R. A., \& Brown, C. R. (1996). Newly qualified primary teachers: A comparative analysis of perceptions held by B. Ed. and PGCE trained teachers of their training routes. Educational Psychology, 16(3), 257-270.

Canter, L., \& M. Canter. 2001. Assertive Discipline: Positive Behavior Management for Today's Classroom. Seal Beach, CA: Canter.

Cho, V., Mansfield, K. C., \& Claughton, J. (2020). The past and future technology in classroom management and school discipline: A systematic review. Teaching and Teacher Education, 90, 103037.

Crocker, L., \& Algina, J. (2006). Introduction to classical and Modern Test Theory. Ohio: Cengage Learning.

Depaepe, F., \& König, J. (2018). General pedagogical knowledge, self-efficacy and instructional practice: Disentangling their relationship in pre-service teacher education. Teaching and Teacher Education, 69, 177-190.

Dicke, T., Elling, J., Schmeck, A., \& Leutner, D. (2015). Reducing reality shock: The effects of classroom management skills training on beginning teachers. Teaching and Teacher Education, $48,1-12$.

Dinçer, Ç., \& Akgün, E. (2015). Okul öncesi öğretmenleri için sınıf yönetimi becerileri ölçeğinin geliştirilmesi ve öğretmenlerin sınıf yönetimi becerilerinin çeşitli değişkenlerle ilişkisi. Eğitim ve Bilim, 40(177).

Dreikurs, R., B. B. Grunwald, \& F. C. Pepper. (1998). Maintaining Sanity in the Classroom: Classroom Management Techniques. Washington, DC: Taylor and Francis.

Early, D. M., Maxwell, K. L., Burchinal, M., Alva, S., Bender, R. H., Bryant, D., ... \& Henry, G. T. (2007). Teachers' education, classroom quality, and young children's academic skills: Results from seven studies of preschool programs. Child Development, 78(2), 558-580.

Early, D. M., Maxwell, K. L., Ponder, B. D., \& Pan, Y. (2017). Improving teacher-child interactions: A randomized controlled trial of making the most of classroom interactions and my teaching partner professional development models. Early Childhood Research Quarterly, 38 , $57-70$.

Emmer, E. T., \& Stough, L. M. (2001). Classroom management: A critical part of educational psychology, with implications for teacher education. Educational psychologist, 36(2), 103-112.

Evertson, C. M., \& Weinstein, C. S. (Eds.). (2013). Handbook of classroom management: Research, practice, and contemporary issues. Routledge. 
Finch, W. H., Bolin, J. E., \& Kelley, K. (2014). Multilevel modeling using R. Boca Raton: Taylor \& Francis Group.

Fraenkel, J. R., Wallen, N. E., \& Hyun, H. H. (2012). How to design and evaluate research in education (8. Ed.). United States: McGraw-Hill Companies.

Gettinger, M. \& Fischer, C. (2014). Early childhood classroom management. In E. T. Emmer \& E. J. Sabornie (Eds.), Handbook of classroom management (pp. 141-166). New York, NY: Routledge.

Glasser, W. 1998. Choice Theory: A New Psychology of Personal Freedom. New York: HarperCollins.

Glock, S. (2016). Stop talking out of turn: The influence of students' gender and ethnicity on preservice teachers' intervention strategies for student misbehavior. Teaching and Teacher Education, 56, 106-114.

Goldstein, H. (2011). Multilevel statistical models. (4th Edition). United Kingdom: John Wiley \& Sons, Ltd.

Gordon, T. 2003. Teacher effectiveness training. New York: Three rivers press.

Güleç, S., P. Bağçeli, \& G. Onur. 2008. “Öğretmen adaylanının sınıf kurallarının belirlenmesi ve uygulanmasına ilişkin görüşleri [Pre-service teachers' opinions about determining and performing classroom rules]. İlkögretim Online [Elementary Education Online] 7 (2), 333-348.

Hamre, B. K., Pianta, R. C., Burchinal, M., Field, S., LoCasale-Crouch, J., Downer, J. T., ... \& ScottLittle, C. (2012). A course on effective teacher-child interactions: Effects on teacher beliefs, knowledge, and observed practice. American Educational Research Journal, 49(1), 88-123.

Harlan, J. C., \& S. T. Rowland. 2002. Behavior management strategies for teachers: achieving instructional effectiveness, student success, and student motivation - Every teacher and every student can. Springfield, IL: Charles C Thomas Publisher.

Hox, J. J. (2010). Multilevel analysis. techniques and applications (Quantitative methodology series) (Second Edition). United States: Routledge.

Hu, B. Y., Dieker, L., Yang, Y., \& Yang, N. (2016). The quality of classroom experiences in Chinese kindergarten classrooms across settings and learning activities: Implications for teacher preparation. Teaching and Teacher Education, 57, 39-50.

Irvine, J. 2015. "Enacting Glasser's (1998) Choice theory in a grade 3 classroom: A Case study. Journal of Case Studies in Education 7, 1-14.

Karademir, A. \& Ören, M. (2020). Okul iklimi: Anaokulu yöneticileri ve öğretmenlerin bakış açısıyla karşılaştırmalı bir araștırma. Eğitimde Nitel Araștırmalar Dergisi - Journal of Qualitative Research in Education, 8(1), 206-236.

Keleş, O. (2015). Okul öncesi öğretmenlerinin sınıf yönetiminde istenmeyen davranışlara karşı kullandığ1 stratejileri belirleme ölçeği geçerlik güvenirlik çalışması. Hacettepe Üniversitesi Sağhlk Bilimleri Fakültesi Dergisi. 
Kokkinos, C. M., Panayiotou, G., \& Davazoglou, A. M. (2004). Perceived seriousness of pupils' undesirable behaviours: The student teachers' perspective. Educational Psychology, 24(1), 109120.

König, J. (2015). Measuring classroom management expertise (CME) of teachers: A video-based assessment approach and statistical results. Cogent Education, 2(1), 991178.

Kwok, A. (2019). Classroom management actions of beginning urban teachers. Urban Education, 54(3), 339-367.

Levine, A. (2006).Educating school teachers. Washington: The Education schools project, Retrieved from.http://www.edschools.org/pdf/Educating_Teachers_Report.pdf.

Levine, B., \& He, Y. (2008). Investigating the content and sources of teacher candidates' personal practical theories (PPTs). Journal of Teacher Education, 59(1), 55-68.

Lippard, C. N., La Paro, K. M., Rouse, H. L., \& Crosby, D. A. (2018). A Closer Look at TeacherChild Relationships and Classroom Emotional Context in Preschool. In Child \& Youth Care Forum (Vol. 47, No. 1, pp. 1-21). Springer US.

Malmgren, K. W., B. J. Trezek, \& P. V. Paul. 2005. Models of classroom management as applied to the secondary classroom. The Clearing House: A Journal of Educational Strategies, Issues and Ideas 79 (1): 36-39.

Martin, N. K., \& D. A. Sass. 2010. Construct validation of the behavior and instructional management scale. Teaching and Teacher Education, 26, 1124-1135.

Martin, S. D. (2004). Finding balance: Impact of classroom management conceptions on developing teacher practice. Teaching and Teacher Education, 20(5), 405-422.

Maskan, A. K. (2007). Preservice science and math teachers' difficulties in disruptive behavior and class management. International Journal of Educational Reform, 16(4), 336-349.

Mikami, A. Y., Griggs, M. S., Reuland, M. M., \& Gregory, A. (2012). Teacher practices as predictors of children's classroom social preference. Journal of School Psychology, 50(1), 95-111.

Ministry of National Education [MoNE] (2013). Pre-school education program.

Neal, S. C., Norwalk, K. E., \& Haskett, M. E. (2020). Differential impacts of the Incredible YearsTeacher Classroom Management program based on young children's risk profiles. Early Childhood Research Quarterly, 51, 473-482.

O’Neill, S., \& Stephenson, J. (2012). Does classroom management coursework influence preservice teachers' perceived preparedness or confidence?. Teaching and Teacher Education, 28(8), 1131-1143.

Oliver, R. M., \& Reschly, D. J. (2007). Effective Classroom management: Teacher preparation and professional development. TQ connection issue paper on improving student outcomes in general and special education. Washington: Retrieved from. https:/ / files.eric.ed.gov/fulltext/ED543769.pdf 
Opdenakker, M. C., \& Van Damme, J. (2006). Teacher characteristics and teaching styles as effectiveness enhancing factors of classroom practice. Teaching and Teacher Education, 22(1), $1-21$.

Osborne, J. W. (2000). The advantages of hierarchical linear modeling. Practical Assessment, Research, and Evaluation, 7(1), 1-4.

Ozmon, H., \& S. M. Craver. 2008. Philosophical Foundations of Education. Upper Saddle River, NJ: Pearson Prentice Hall.

Öztürk, Y., Gangal, M., \& Ergişi, M. B. (2014). Okul öncesi öğretmen adaylarının eğitimlerinin sınıf yönetimi ve stratejileri üzerindeki etkisine ilişkin görüşlerinin incelenmesi. Eržincan Üniversitesi Ë̆itim Fakültesi Dergisi, 16(1), 224-238.

Parpucu, N., Yıldırım-Polat, A., \& Akman, B. (2018). Okul öncesi öğretmenlerinin sınıf yönetiminde ilişkiler ve iletişime yönelik görüşleri. Journal of Theory \& Practice in Education (JTPE), 14(4), 417-433.

Pianta, R. C. (2006). Classroom management and relationships between children and teachers: implications for research and practice. In C. M. Evertson, \& C. S.Weinstein (Eds.), Handbook of classroom management, research, practice and contemporary issues (pp. 685e709). Mahwah, NJ: Erlbaum.

Pianta, R. C., Mashburn, A. J., Downer, J. T., Hamre, B. K., \& Justice, L. (2008). Effects of webmediated professional development resources on teacher-child interactions in prekindergarten classrooms. Early Childhood Research Quarterly, 23(4), 431-451.

Quieng, M. C., Lim, P. P., \& Lucas, M. R. D. (2015). 21st Century-based soft skills: Spotlight on Non-cognitive skills in a Cognitive-Laden Dentistry Program. European Journal of Contemporary Education, 11(1), 72-81.

Rathel, J. M., Drasgow, E., \& Christle, C. C. (2008). Effects of supervisor performance feedback on increasing preservice teachers' positive communication behaviors with students with emotional and behavioral disorders. Journal of Emotional and Behavioral Disorders, 16(2), 67-77.

Raudenbush, S. W., \& Bryk, A. S. (2002). Hierarchical linear models: Applications and data analysis methods. London: Sage.

Reupert, A., \& Woodcock, S. (2010). Success and near misses: Pre-service teachers' use, confidence and success in various classroom management strategies. Teaching and Teacher Education, 26, 1261-1268.

Şahin-Sak, İ. T., Sak, R., \& Tezel-Şahin, F. (2018). Preschool teachers' views about classroom management models. Early Years, 38(1), 35-52.

Sak, İ. Ş. (2015). Okul öncesi öğretmen adaylarının sınıf yönetimi ile ilgili öz-yeterlik inançları. Sakarya Üniversitesi Ë̆itim Fakültesi Dergisi, 29, 101-120.

Sak, R., Şahin Sak, İ. T., \& Yerlikaya, İ. (2015). Behavior management strategies: Beliefs and practices of male and female early childhood teachers. European Early Childhood Education Research Journal, 23(3), 328-339. 
Salkovsky, M., \& Romi, S. (2015). Teachers' coping styles and factors inhibiting teachers' preferred classroom management practice. Teaching and Teacher Education, 48, 56-65.

Shawer, S. F. (2017). Teacher-driven curriculum development at the classroom level: Implications for curriculum, pedagogy and teacher training. Teaching and Teacher Education, 63, 296-313.

Siebert, C. J. (2005). Promoting preservice teachers' success in classroom management by leveraging a local union's resources. A professional development school initiative. Education, 125(3), 385-392.

Soheili, F., H. Alizadeh, J. M. Murphy, H. S. Bajestani, \& E. D. Ferguson, 2015. "Teachers as Leaders: The Impact of Adler-Dreikurs classroom management techniques on students' perceptions of the classroom environment and on academic achievement." The Journal of Individual Psychology 71 (4): 440-461.

Straub, C., Dieker, L., Hynes, M., \& Hughes, C. (2014). Using virtual rehearsal in TLE TeachLivETM mixed reality classroom simulator to determine the effects on the performance of mathematics teachers. Teach Live National Research Project: Year 1 Findings, $1-50$.

Sullivan, G. M., \& Feinn, R. (2012). Using Effect Size - or Why the P Value Is Not Enough. Journal of Graduate Medical Education, 4(3), 279-282.

Sun, R. (2015). Teachers' experiences of effective strategies for managing classroom misbehavior in Hong Kong. Teaching and Teacher Education, 46, 94-103.

Swabey, K., Castleton, G., \& Penney, D. (2010). Meeting the standards? Exploring preparedness for teaching. Australian Journal of Teacher Education, 35(8), 3.

Talvio, M., K. Lonka, E. Komulainen, M. Kuusela \& T. Lintunen. (2013). Revisiting Gordon's teacher effectiveness training: An Intervention study on teachers' social and emotional learning. Electronic Journal of Research in Educational Psychology 11(3), 693-716.

Toran, M. \& Gençgel Akkuş, H. (2016). Okul öncesi öğretmenlerinin sınıf yönetimi becerilerinin değerlendirilmesi: KKTC Örneği. Kastamonu Ë̆itim Dergisi, 24(4), 2041-2056.

Türk, C., Kartal, A., Karademir, A. \& Öcal, E. (2019). Preschool teacherse views of classroom management processes. Uluslararası Türkȩe Edebiyat Kültür Eğitim Dergisi, 8(4), 2282-2299.

Von Secker, C. E., \& Lissitz, R. W. (1999). Estimating the impact of instructional practices on student achievement in science. Journal of Research in Science Teaching, 36(10), 1110-1126.

Wang, J., Xie, H., \& Fisher, J. H. (2012). Multilevel models: Application using SAS. Germany: De Gruyter.

Weber, K. E., Gold, B., Prilop, C. N., \& Kleinknecht, M. (2018). Promoting pre-service teachers' professional vision of classroom management during practical school training: Effects of a structured online-and video-based self-reflection and feedback intervention. Teaching and Teacher Education, 76, 39-49. 
Wolff, C. E., Jarodzka, H., \& Boshuizen, H. P. (2017). See and tell: Differences between expert and novice teachers' interpretations of problematic classroom management events. Teaching and Teacher Education, 66, 295-308.

Wolfgang, C. H. 1996. The Three faces of discipline for the elementary school teacher: Empowering the teacher and students. Boston, MA: Allyn and Bacon.

Woltman, H., Feldstain, A., MacKay, J. C., \& Rocchi, M. (2012). An introduction to Hierarchical Linear Modeling. Tutorials in Quantitative Methods for Psychology, 8(1), 52-69.

Yumuş, M., \& Bayhan, P. (2017). Early childhood behavioural problems in Turkey: teachers' views, challenges and coping strategies. Early Child Development and Care, 187(12), 1833-1843.

Zembat, R., Tunçeli, H. ̇̇., \& Akşin Yavuz, E. (2017). Okul öncesi öğretmenlerinin sınıf yönetimi becerileri ile problem çözme becerileri arasındaki ilişkinin incelenmesi. Abi Evran Üniversitesi Kirşehir Ë̆itim Fakülttesi Dergisi, 18(3), 24-43. 